\title{
Declining of Sensing Errors in Cognitive Radio by Threshold Determination of Energy Spotting Method
}

\author{
M. Ajay Kumar, N.Rajesha, Anupama Deshpande
}

\begin{abstract}
The conventional Cognitive Radio Network is anticipates the fundamental solution to problem of spectrum scarcity in the forthcoming wireless and cellular communication networks, furthermore CR networking senses vacant spectrum bands in opportunistic method, and though, increases the effectiveness of spectrum usage. The CRN (Cognitive radio Network) provides the wireless connectivity and integration through the medium of dynamic spectrum access approaches and heterogeneous wireless designs and architectures. The enabling techniques and utilities of the CRNs have received the dynamic research and the industry interest. In this specific paper, we proposed an solution to decrease the error rate by enhancing the performance of detection which occur in time of spectrum sensing mechanism.
\end{abstract}

Keywords- Cognitive Radio, Spectrum Sensing, Vacant Frequency Band, Spectrum Utilization.

\section{INTRODUCTION}

The cognitive radios one of effective future wireless communication technology which exploits the opportunistic and dynamic spectrum utilization strategies to determine with wireless and cellular connectivity. The CRNs are premeditated as vital technology to mitigate the typical spectrum under usage as well as there is a need of solution for the scarcity of spectrum and the permissive component of the conventional $\mathrm{CR}$ and the dynamic spectrum accessibility in cognitive radio (CR). The $\mathrm{CR}$ is intelligent wireless device which detects the neighboring radio conditions moreover opportunistically use vacant spectrum bands dependent on activities of neighboring initial licensed networks and the comprehensive research efforts has carried on distinct ideas as well as the CR design over the decades [4]. However, the energy efficiency of CRNs has received far less interest.

Cognitive radio is going to be a very assuring solution to reduce this scarcity and inadequacy in radio frequency spectrum. CRNs promises to enlarge the usage of spectrum by using the idle bands and utilizing it for purpose of communication. The Spectrum-sensing is vital function to detect these vacant spectral bands [3]. Furthermore to sense the spectrum bands there are effective spectrum sensing approaches such as matched filter approach, energy spotting method. There is much research is going on advantages and

Revised Manuscript Received on July 10, 2019.

M. Ajay Kumar, Research Scholar, Dept of ECE, Shri JJT University, Jhunjhunu-01, Rajasthan, India. (ajayajju126@gmail.com)

N.Rajesha, Dept of ECE, Malla Reddy Institute of Engg and Tech, Hyderabad-14, A.P, India (rajeshmurthy44@gmail.com)

Anupama Deshpande, Dept of ECE, Shri JJT University, Jhunjhunu01 Rajasthan, India (Mangala.d.2000@gmail.com) drawbacks of spectrum-sensing mechanism. The energy spotting dependent spectrum sensing approach is an effective technique because of simplicity and lower computational difficulty and simplicity, and also no priorknowledge regarding the signal attributes to be sensed is necessary and essential.

Distinct functional and operational essentials are to be considered while deploying the $\mathrm{CRN}$, alike as no intervention/interference to primary-user. This must essential of higher probability of detection probability $\left(\mathrm{P}_{\mathrm{dt}}\right)$ or to increase the CRN throughput, that need of lower falsealarm probability $\left(\mathrm{P}_{\mathrm{fa}}\right)$. In energy spotting approach, the both probabilities involve the tradeoff by that the overall probability regarding the errors remain higher.

In modern times, there is numerous investigations are conducting in virtue to maximize the performance of energy-spotting performance. The typical Energy spotting method is majorly dependent on the fixed threshold that may not effectively work in lower SNR cases. It is for the sack; the cognitive radio performance is based on the received SNR and the hidden initial terminal issue and uncertainty because of shadowing and fading are the main reasons of the received signal decline at CRNs terminal.

This issue can be resolved by the co-operative sensing methods by exploit of inherent and instinctive spatial diversity. Nevertheless these sensing techniques are depends on the noise power assumptions.

Virtually the noise-power alters with both location and time of terminal that is major cause of un-certain noise. The major focus of this investigative work is to minimize noise uncertainty impact on the signal detection and to decrease the sensing errors by adopting threshold regulations In this we proposed the optimal threshold model that dependent on assumptions of noise power and by that probability of sensing errors be minimized which depends on depends false alarm model by which it increase the spectrum utilization, moreover it ensures the lower interference to cognitive PUs.

\section{SYSTEM MODEL}

Consider the CR terminal which essential to sense the presence or absence of cognitive primary-user signal by adopting the energy spotting method. In this approach the
Blue Eyes Intelligence Engineering $\&$ Sciences Publication 


\section{DECLINING OF SENSING ERRORS IN COGNITIVE RADIO BY THRESHOLD DETERMINATION OF ENERGY SPOTTING METHOD}

PU's transmitted power will sensed firstly and then correlates with premeditated threshold to define whether the frequency band is accessible or not.

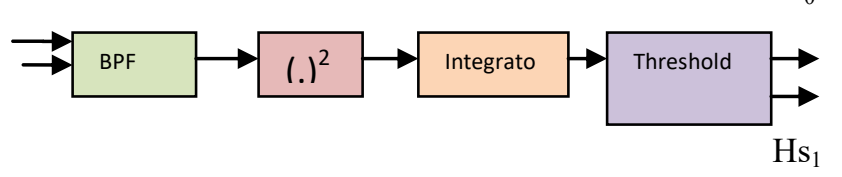

Fig 1: System model of Energy Spotting Method

The above figure is energy spotting method system model; the band-pass filter eliminates the unwanted band signals and allows only certain range which required after this the signal has digitalized by analog-to-digital convertor, and then squared as well as averaged to measure the received-signal energy. This received signal's measured energy is analyzed with the threshold to determine either the spectrum band is accessible or not. By this we can determine the hypothesis s follows:

$$
\begin{array}{ll}
q(n)=p(n) & \mathrm{Hs}_{0} \\
q(n)=p(n)+r(n) & H s_{1}
\end{array}
$$

Whereas $\mathrm{Hs}_{0}$ is the hypothesis which PU is not using its frequency band and $\mathrm{Hs}_{1}$ is the hypothesis which cognitive PU using the frequency band for its act of transmission, $q(n)$ is received-signal at cognitive secondary-user; $p(n)$ is the AWGN (Additive White Gaussian Noise) sample having the variance $\sigma_{n}^{2}$. The hypotheses $H s_{0}$ say about the primary user unavailability and the $H \mathrm{~s}_{1}$ represents about PUs availability. The noise samples are relevant to variance as specified:

$$
\mathrm{p}(\mathrm{n}) \in \mathrm{N}\left(0, \sigma_{n}^{2}\right)
$$

In case, the spectrum-sensing is quite fails, in such conditions the resulting possibility is referred as false alarm probability and missed detection probability. In conditions of missed detection probability, when the CR primary-user is in availability in the frequency band then typically the mechanism of the spectrum sensing opt the hypotheses $\mathrm{Hs}_{0}$. In scenario of false alarm possibility, when the band is unused/vacant then the method opts hypotheses $\mathrm{Hs}_{1}$. In the missed-detection probability, there is the reaction of resulting the interference to cognitive primary-user. At the same time in false alarm probability results in the missing of opportunities as well as due to the which spectrum utilization gradually decreases in efficient manner and it can conclude with two of the probabilities particularly, probability of false alarm and detection. For, the optimum performance of $\mathrm{CR}$; it expected as detection probability must enlarged with the decrease of false alarm possibility.

The detection probability, $\mathrm{P}_{\mathrm{dt}}=\mathrm{r}\left(\mathrm{Hs}_{1} / \mathrm{Hs}_{1}\right)$ and

The false alarm probability, $\mathrm{P}_{\mathrm{fa}}=\operatorname{Pr}\left(\mathrm{Hs}_{1} / \mathrm{Hs}_{0}\right)$

The test statistic's is defined as:

$$
\mathrm{Z}(\mathrm{y})=\sum_{n=0}^{N} q(n)^{2}
$$

Whereas, the $\mathrm{q}(\mathrm{n})$ represents primary signal's received energy, $\mathrm{Z}(\mathrm{y})$ is decision-variable and the test static's follows the central-distribution chi square below the hypotheses $\mathrm{Hs}_{0}$ as well as the non-central distribution below the hypotheses $\mathrm{Hs}_{1}$. Although in consideration of region having lower SNR, the number of samples essential to gain the performance in the detection and false alarm probability should be more than the single sample, moreover the Gaussian distribution and Central Limit Theorem has adopted to determine the test-statistic's.

The detection \& false alarm probabilities through the AWGN-channel are illustrated respectively has below:

$$
\mathbf{P}_{\mathrm{dt}}=\operatorname{Prob}\left(\mathrm{Z}\left(\mathrm{q}>\lambda_{d t} \mid H s_{1}\right)\right)
$$

$$
\begin{gathered}
\mathbf{P}_{\mathrm{dt}}=\frac{1}{2}\left(\frac{\lambda_{d t}-\varepsilon_{1}}{\rho_{1}}\right) \\
\mathbf{P}_{\mathrm{fa}}=\operatorname{Prob}\left(\mathrm{Z}\left(\mathrm{q}>\lambda_{f a} \mid H s_{0}\right)\right) \\
\mathbf{P}_{\mathrm{fa}}=\frac{1}{2}\left(\frac{\lambda_{f a}-\varepsilon_{0}}{\rho_{0}}\right)
\end{gathered}
$$

There is the other vital probability criterion referred as miss detection probability $\mathrm{P}_{\mathrm{md}}$. The energy spotter represents the absence of primary user, while the cognitive $\mathrm{PU}$ is actually in presence mode.

$$
\mathbf{P}_{\text {md }}=\mathbf{1 -} \mathbf{P}_{\mathrm{dt}}
$$

In such scenarios; we can systematically specify the sensing error probability as follow:

$$
\mathrm{P}_{\mathrm{er}}=\mathrm{PHs}_{0} \mathrm{P}_{\mathrm{fa}}+\mathrm{PHs}_{1} \mathrm{P}_{\mathrm{md}}
$$

$\mathrm{PHs}_{0} \mathrm{Pfa}$ represents the absent of primary user, while the energy spotter indicates the presence of primary user. $\mathrm{PHs}_{1}$ $\mathrm{P}_{\mathrm{md}}$ illustrates the presence of primary-user; while the energy spotter representing the absence of primary user. Furthermore, it is to referral as $\mathrm{PHs}_{1}+\mathrm{PHs}_{0}=1$.

\section{POSSIBILITY OF ERROR (PER) WITH STABLE RATE OF FALSE ALARM}

The performance of spectrum sensing rely upon the sensing error probability, that eventually be minimized. In mechanism of spectrum sensing procedure, it is necessary to decline the false alarm possibility to access the better opportunities of spectrum.

Moreover, the Low miss-detection probability provides protection and security to CR primary users. In this specific segment, the sensing error probability is the threshold function characterized by keeping the constant rate of false alarm (CFAR) by assuming that the noise is known fully. In these scenario the threshold is attained from the Eq (6) and also defined by the Eq (9)

$$
\lambda_{f a}=\operatorname{Erfcinv}\left(2 \mathrm{P}_{\mathrm{fa}}\right) * \sqrt{2} \rho_{0}+\varepsilon_{0}
$$

To prevent the notational difficulty; we notice the threshold attained from Eq (6) as the $\lambda_{f a}$.

\section{ERROR POSSIBILITY WITH STABLE DETECTION RATE}

In this particular segment, we examine the error probability as threshold defined function by constant detection rate (CDR) by presuming fully known noise. In these specific cases the threshold as accessed from the Eqn. (5) as characterized by 


$$
\lambda_{d t}=\operatorname{Erfcinv}\left(2 \mathrm{P}_{\mathrm{dt}}\right) * \sqrt{2} \rho_{1}+\varepsilon_{1}(10)
$$

Attained threshold from the Eq (5) is noticed as $\lambda_{d t}$ and higher detection possibilities ensure the protection to PU.

\section{ERROR PROBABILITY UNDER THE NOISE- UNCERTAINTY}

Basically there are certain reasons for noise in faded received signal and by autonomous sources the noise may caused due to diffractions, reflections and scattering etc. Nevertheless the power of noise level can vary over location and time, which makes uncertainty of noise. In virtue to analyze noise uncertainty effect on performance of detection, we concede that $\boldsymbol{\rho}_{\boldsymbol{n}}^{2}$ is nominal noise uncertainty and noise power is admitted as existent with-in range $\rho^{2}=\left(\rho_{\min }^{2}, \rho_{\max }^{2}\right)$ whereas $\rho^{2}$ is noise power that estimated, $\rho_{\text {min }}^{2}=\left(\frac{\rho_{n}^{2}}{a}\right)$ is noise uncertainties lower limit, $\rho_{\text {max }}^{2}=a \rho_{n}^{2}$ characterizes noise uncertainty upper limit, $(\mathrm{a} \geq$ $1)$ is factor of noise uncertainty. Depending on the theorem of central limit [5], and the test statistics for the noise be proximate as the Gaussian-distribution and represented as subsequent

$$
\begin{aligned}
\mathrm{Z}(\mathrm{y}) & =\left\{\begin{array}{c}
\text { Normal }\left(N a \rho_{n, 2}^{2} 2 N a^{2} \rho_{n}^{4}\right) \\
\text { Normal }\left(N \rho_{n}^{2}\left(\frac{1}{a}+\gamma\right), 2 N \rho_{n}^{4}\left(\frac{1}{a}+\gamma\right)\right)
\end{array}\right. \\
P_{d t} & =\frac{1}{2} \operatorname{erfc}\left[\frac{\lambda_{d t}-N \rho_{n}^{2}\left(\frac{1}{a}+\gamma\right)}{\sqrt{4 N} \rho_{n}^{2}\left(\frac{1}{a}+\gamma\right)}\right] \\
P_{f a} & =\frac{1}{2} \operatorname{erfc}\left[\frac{\lambda_{f a}-N a \rho_{n}^{2}}{\sqrt{4 N} a \rho_{n}^{2}}\right]
\end{aligned}
$$

\section{SIMUTATION OUTPUTS AND ANALYSIS RESULTS}

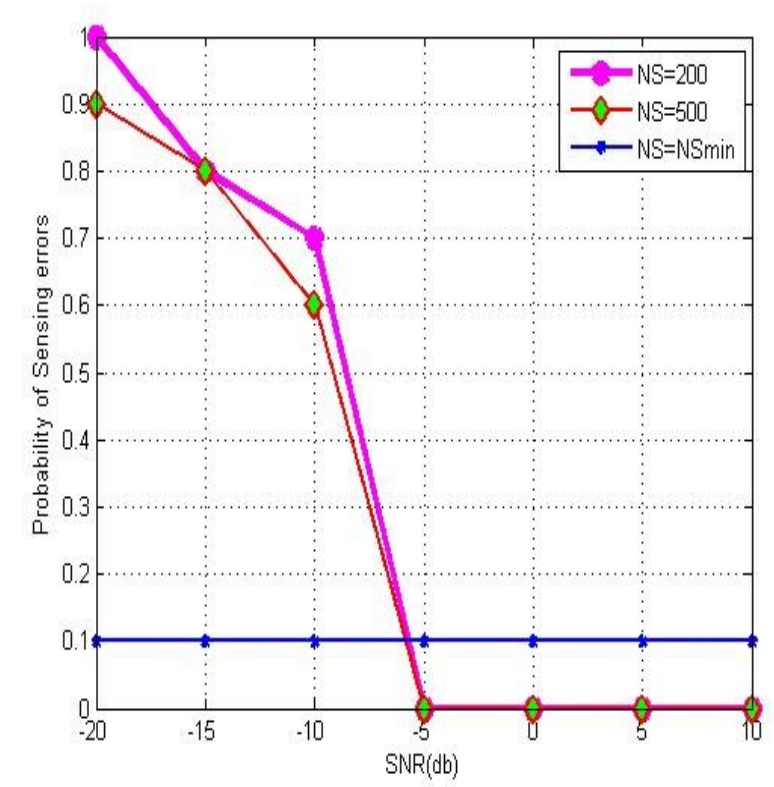

Fig 2: SNR VS Error probability

The figure 2 interprets the correlation among the signal to noise ratio and error probability by taking the sum of samples. At $\mathrm{N}=200$ and 500 there is maximum error at lower SNR and when the SNR is quite increasing the error possibility is decreases at high SNR the error anticipation is insignificant, furthermore when sum of samples is equal to minimal samples the error possibility is less for lower and higher SNR.

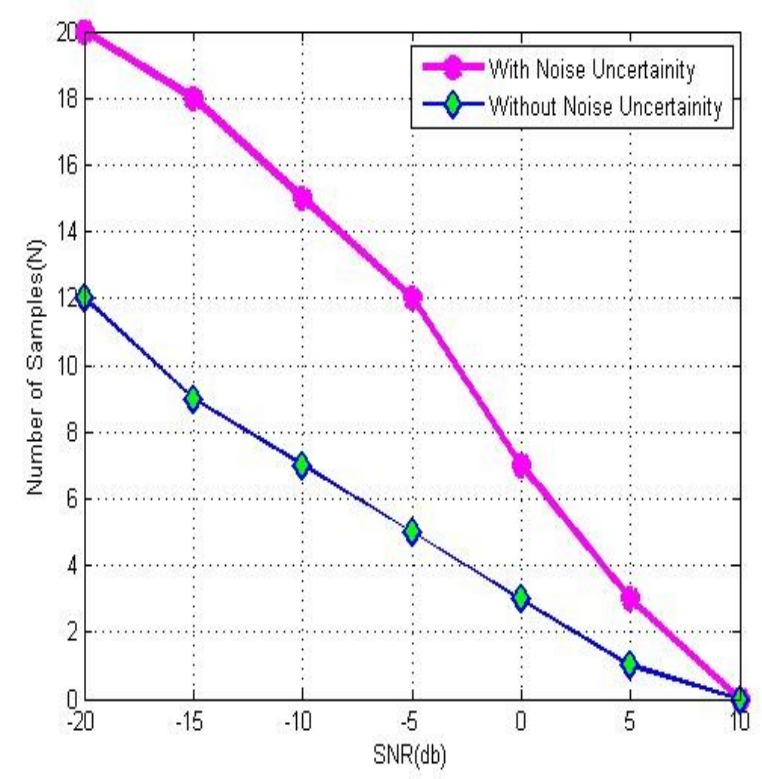

Fig 3: Effect of noise uncertainty at different SNR

The above shows the interrelation between SNR as well as number of samples in existence and absence of uncertainty of noise, the uncertainty of noise leads to detection failure and result in spectrum inefficiency.

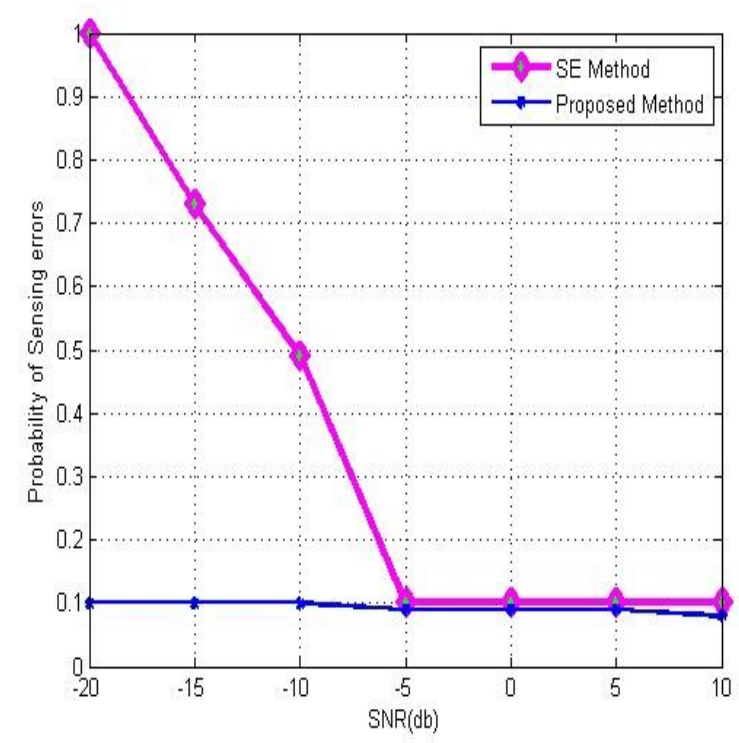

Fig 3: Proposed method comparison with method of Sensing Error

The Figure 3 clearly shows the sensing errors with various SNR ranges, it also clearly compared the proposed method with sensing error method, we can observe that in proposed method the sensing error possibility is around 0.1 for higher and lower SNR and more effective than the SE method which can increase the usage of spectrum efficiently. 


\section{DECLINING OF SENSING ERRORS IN COGNITIVE RADIO BY THRESHOLD DETERMINATION OF ENERGY SPOTTING METHOD}

\section{CONCLUSION}

This research work mainly focuses on the reducing the possibility of sensing the error in different SNR ranges with or without noise uncertainty to improve the spectrum utilization by taking the number of samples and by consolidating through distinct lower and higher SNR, moreover the spectrum sensing methods clearly gives the status of CR users are in neither presence nor absence by allocate the vacant band to the other user and also by adopting the energy spotting technique we achieved the 0.1 error probability in proposed method which increases spectrum efficiency.

\section{REFERENCES}

1. V Amrutha, K.V. Karthikeyan, "Spectrum sensing methodologies in cognitive radio networks: A survey" in communications IEEE 3-4 Feb. 2017.

2. M. Meena, F. Bhagari, V. Rajendran, "Spectrum Sensing Using Cognitive Radio Technology" " in communications; IEEE 6-8 April 2017.

3. Ravi Verma, Arunanshu Mahapatro, "Cognitive Radio: Energy detection using wavelet packet transform for spectrum sensing" in communications; IEEE 27-28 Feb. 2017.

4. J. Avila; R. Subashree, "Improved method of sensing the spectrum holes in cognitive radio" in communications; IEEE 5-7 Jan. 2017.

5. Euler Edson Apaza Medina; Silvio Ernesto Barbin, "Performance of Spectrum Sensing Based on Energy Detection for Cognitive Radios" in communications; IEEE 10-14 Sept. 2018.

6. M.Ajay Kumar \& Dr.N.Rajesha "Effective Analysis on Matched Filter Technique in Cognitive Radio", IJAER, Volume 14, Number 3 (2019) pp. 840-844.

7. G. Shine Let; G. Josemin Bala; C. Benin Pratap, "Comparative study of heuristic approaches used for spectrum sensing in cognitive radio network" " in communications; IEEE 19-20 Jan. 2018.

8. M.Ajay Kumar \& Dr.N.Rajesha "Performance Enhancement of Effective Spectrum Sensing Approaches in Cognitive Radio", 2019 JETIR March 2019, Volume 6.

9. Peng Cheng, Z. Zhang, H. Huang and P. Qiu, “ A Distributed Algorithm for Optimal Resource Allocation in Cognitive OFDMA Systems", in Proc. IEEE Int. Conf. Communications (ICC), May 2008, pp.4718- 4723.

10. Hossan M Farag \& Enab Mohamad 2014, Improved Cognitive Radio Energy Spotting Algorithm Based upon Noise Uncertainty Estimation, IEEE International Conference, pp.44-51.

11. Dongho Seo, Haewoon Nam, "A Parallel Multi-Channel Cooperative Spectrum Sensing in Cognitive Radio Networks" in communications; IEEE 23-26 Oct. 2018.

12. M.Ajay Kumar, Dr,N.Rajesha \& Dr.Anupama Deshpande "Attainable Throughput Determination for Cognitive Radio", IJRAT Vol.7, No.4, April 2019.

13. Pitcha Rungsawang, Amnach Khawne, "The implementation of spectrum sensing and spectrum allocation on cognitive radio" in communications; IEEE 19-22 Feb. 2017.

14. B. Wang and K. Liu," Advances in Cognitive radio networks: A survey," Selected Topics in Signal Processing, IEEE Journal, 5(1)(2011) 5-23.

15. Ahmad Ali Tabassam, Mohammad Uzair Suleman, Sunil Kalasit and Shehyar Khan 2011, Building Cognitive Radios in MATLAB Simulink - A Step Towards Future Wireless Technology, IEEE 2011.

16. "Matched Filter Spotting with Dynamic Threshold for Cognitive Radio Networks", proceedings in IEEE 2015 International Conference on Wireless Networks and Mobile Communications (WINCOM).
17. Binshen, Longyang Huang and Chengshi Zhao 2008, Energy Spotting Based Spectrum sensing for Cognitive Radios in Noise of uncertainty, IEEE International Conference,pp.1218. 